\title{
SPITZER OBSERVATIONS OF GJ 3470 b: A VERY LOW-DENSITY NEPTUNE-SIZE PLANET ORBITING A METAL-RICH M DWARF*
}

\author{
Brice-Olivier Demory ${ }^{1}$, Guillermo Torres $^{2}$, VAsco Neves $^{3,4,5}$, Leslie Rogers $^{6}$, Michaël Gillon $^{7}$, Elliott Horch $^{8,12}$, \\ Peter Sullivan $^{9}$, Xavier Bonfils $^{5}$, Xavier Delfosse $^{5}$, Thierry Forveille ${ }^{5}$, Christophe Lovis $^{10}$, Michel Mayor $^{10}$, \\ Nuno Santos ${ }^{3,4}$, Sara Seager ${ }^{1,9}$, Barry Smalley ${ }^{11}$, and Stephane Udry ${ }^{10}$ \\ ${ }^{1}$ Department of Earth, Atmospheric and Planetary Sciences, Massachusetts Institute of Technology, \\ 77 Massachusetts Ave., Cambridge, MA 02139, USA; demory@mit.edu \\ ${ }^{2}$ Harvard-Smithsonian Center for Astrophysics, 60 Garden St., Cambridge, MA 02138, USA \\ ${ }^{3}$ Centro de Astrofísica, Universidade do Porto, Rua das Estrelas, 4150-762 Porto, Portugal \\ ${ }^{4}$ Departamento de Física e Astronomia, Faculdade de Ciências, Universidade do Porto, Rua do Campo Alegre, 4169-007 Porto, Portugal \\ ${ }^{5}$ UJF-Grenoble 1/CNRS-INSU, Institut de Planétologie et d'Astrophysique de Grenoble (IPAG) UMR 5274, Grenoble, F-38041, France \\ ${ }^{6}$ Department of Astrophysics, California Institute of Technology, MC 249-17, Pasadena, CA 91125, USA \\ ${ }^{7}$ Institut d'Astrophysique et de Géophysique, Université de Liège, Allée du 6 Août, 17, Bat. B5C, Liège 1, Belgium \\ ${ }^{8}$ Department of Physics, 501 Crescent Street, Southern Connecticut State University, New Haven, CT 06515, USA \\ ${ }^{9}$ Department of Physics and Kavli Institute for Astrophysics and Space Research, MIT, 77 Massachusetts Avenue, Cambridge, MA 02138, USA \\ ${ }^{10}$ Observatoire de Genève, Université de Genève, $51 \mathrm{ch}$. des Maillettes, CH-1290 Versoix, Switzerland \\ ${ }_{11}$ Astrophysics Group, Keele University, Staffordshire, ST55BG, UK \\ Received 2013 January 28; accepted 2013 March 4; published 2013 April 25
}

\begin{abstract}
We present Spitzer/IRAC $4.5 \mu \mathrm{m}$ transit photometry of GJ $3470 \mathrm{~b}$, a Neptune-size planet orbiting an M1.5 dwarf star with a 3.3 day period recently discovered in the course of the HARPS M-dwarf survey. We refine the stellar parameters by employing purely empirical mass-luminosity and surface brightness relations constrained by our updated value for the mean stellar density, and additional information from new near-infrared spectroscopic observations. We derive a stellar mass of $M_{\star}=0.539_{-0.043}^{+0.047} M_{\odot}$ and a radius of $R_{\star}=0.568_{-0.031}^{+0.037} R_{\odot}$. We determine the host star of GJ $3470 \mathrm{~b}$ to be metal-rich, with a metallicity of $[\mathrm{Fe} / \mathrm{H}]=+0.20 \pm 0.10$ and an effective temperature of $T_{\text {eff }}=3600 \pm 100 \mathrm{~K}$. The revised stellar parameters yield a planetary radius $R_{p}=4.83_{-0.21}^{+0.22} R_{\oplus}$ that is $13 \%$ larger than the value previously reported in the literature. We find a planetary mass $M_{p}=13.9_{-1.4}^{+1.5} M_{\oplus}$ that translates to a very low planetary density, $\rho_{p}=0.72_{-0.12}^{+0.13} \mathrm{~g} \mathrm{~cm}^{-3}$, which is $33 \%$ smaller than the original value. With a mean density half of that of GJ 436 b, GJ $3470 \mathrm{~b}$ is an example of a very low-density low-mass planet, similar to Kepler-11 d, Kepler-11 e, and Kepler-18 c, but orbiting a much brighter nearby star that is more conducive to follow-up studies.
\end{abstract}

Key words: planetary systems - stars: individual (GJ 3470) - techniques: photometric - techniques: spectroscopic

Online-only material: color figures

\section{INTRODUCTION}

In the regime of low mass exoplanets only a handful of those known to periodically pass in front of their host stars have transits that are deep enough and orbit parent stars that are bright enough to make them amenable to extensive followup observations. The Kepler mission has recently announced a harvest of more than 2700 planetary candidates identified since the launch of the spacecraft in 2009 (Batalha et al. 2013). About $10 \%$ are Jupiter-size planets with radii between 0.7 and 2.0 Jupiter radii, while more than $55 \%$ are Neptune-size planets with radii between 2 and 6 Earth radii. On the other hand, among the 241 confirmed transiting exoplanets (coming mainly from ground-based surveys), $62 \%$ are Jupiter-size planets with radii between 0.7 and 2.0 Jupiter radii. ${ }^{13}$ It is now clear from Kepler and other studies that short-period Jupiter-size objects make up a relatively small fraction of the exoplanet population

\footnotetext{
* This paper includes data gathered with the $6.5 \mathrm{~m}$ Magellan Telescopes located at Las Campanas Observatory, Chile.

12 Visiting Astronomer, Kitt Peak National Observatory, National Optical Astronomy Observatories, which is operated by the Association of Universities for Research in Astronomy (AURA), Inc., under cooperative agreement with the National Science Foundation.

${ }^{13}$ Source: http://www.exoplanets.org
}

(e.g., Howard et al. 2010, 2012; Wittenmyer et al. 2011). This stark contrast between confirmed exoplanets and the large underlying population glimpsed by Kepler has motivated intense efforts toward the characterization of smaller planets, in order to reach a comparable state of knowledge to what has been learned about the hot-Jupiter population. These efforts already began several years ago with the launch of a number of groundbased projects dedicated to M-dwarf monitoring using both spectroscopy (e.g., the HARPS program; Bonfils et al. 2013) and photometry (e.g., MEarth; Nutzman \& Charbonneau 2008). Planets orbiting M-dwarf stars offer the possibility to probe smaller planets for a given transit depth, because of the favorable star-to-planet radius ratio. GJ 436 b (Butler et al. 2004; Gillon et al. 2007) and GJ $1214 \mathrm{~b}$ (Charbonneau et al. 2009) are the smallest planets orbiting M stars with $K$ magnitude brighter than nine, enabling detailed follow-up studies both from the ground and from space (e.g., Stevenson et al. 2010; Bean et al. 2010).

In the Neptune-mass range, thanks to its relatively large transit depth and host star brightness, GJ 436 b remains a "Rosetta stone" for our understanding of a whole class of exoplanets, shown to be ubiquitous in our Galaxy. With a mass 22 times that of Earth and a radius 4 times larger than our home planet, GJ 436 $\mathrm{b}$ has a relatively high density $\left(\rho_{p}=1.69_{-0.12}^{+0.14} \mathrm{~g} \mathrm{~cm}^{-3}\right.$; Torres et al. 2008), suggesting the presence of a massive core made of 
silicates and/or ices. However, a $\mathrm{H} / \mathrm{He}$ envelope is needed to reproduce its observed radius (e.g., Figueira et al. 2009; Rogers \& Seager 2010a). The improvement in the planetary radius of this object brought about by Spitzer observations placed significant constraints on the range of possible compositions of GJ 436 b's interior. A key question that still needs to be addressed, however, is the extent to which GJ $436 \mathrm{~b}$ is representative of the entire exo-Neptune population.

GJ 3470 b is a new transiting Neptune-size planet discovered in the past year (Bonfils et al. 2012). It orbits a $K_{s}=7.99 \mathrm{mag}$, M1.5 dwarf with a period of 3.337 days. With a published mass of $14.0 \pm 1.7$ Earth masses and a radius of $4.2 \pm 0.6$ Earth radii (Bonfils et al. 2012), GJ 3470 b has a mean density $\rho_{p}=1.07 \pm 0.43 \mathrm{~g} \mathrm{~cm}^{-3}$ that is significantly smaller than that of GJ 436 b. The Kepler mission confirmed several of these so-called "low-density Neptunes." The first two were Kepler-11 d and e (Lissauer et al. 2011), both belonging to the most populated transiting planet system known to date, and the third was Kepler-18 c (Cochran et al. 2011), also a member of a multi-planet system. These objects represent the tip of the iceberg, as several hundred Neptune-size planet candidates have already been detected by Kepler and await confirmation. Unfortunately, most of these Kepler planets orbit faint stars and exhibit shallow transit depths that render follow-up studies very challenging, if not impractical altogether. Aside from Kepler, the ground-based survey HAT discovered the low-density Neptune HAT-P-26 b (Hartman et al. 2011) which, until the discovery of GJ $3470 \mathrm{~b}$, represented the most promising target for follow-up studies. However, as compared to GJ 3470 b, the smaller planetto-star area ratio coupled with the lower brightness of its larger K1 host $\operatorname{star}(K=9.6)$ makes HAT-P-26 b a less favorable target for follow-up studies. GJ $3470 \mathrm{~b}$ therefore presents an ideal opportunity to investigate the internal structure, atmospheric composition, and possible formation pathways of low-density Neptune-size planets (e.g., Rogers et al. 2011).

All transit photometry available so far for GJ 3470 has been collected from the ground. While these time series confirm the transiting nature of GJ $3470 \mathrm{~b}$, they do not precisely constrain the transit parameters, resulting in poorly determined planetary properties. We present in this paper the analysis of two transits of GJ 3470 b obtained with the Spitzer Space Telescope at $4.5 \mu \mathrm{m}$ in the frame of our DDT program (Demory et al. 2012a), submitted shortly after GJ 3470 b's discovery. These data yield a significant refinement of the system parameters. The paper is organized as follows. Section 2 describes the observations and data reduction, while Section 3 presents the photometric and spectroscopic data analyses. Section 4 is dedicated to the stellar characterization, and the resulting planetary parameters are reported in Section 5. We discuss GJ 3470 b's internal structure and composition in Section 6.

\section{OBSERVATIONS AND DATA REDUCTION}

\subsection{Spitzer IRAC $4.5 \mu \mathrm{m}$ Photometry}

We observed two consecutive transits of GJ $3470 \mathrm{~b}$ at $4.5 \mu \mathrm{m}$ using Spitzer's InfraRed Array Camera (IRAC; Fazio et al. 2004). Observations took place on 2012 June 11 and 15 UTC as part of our DDT program PID 80261. For each transit we obtained 780 sets of 64 subarray frames each, with an exposure time of $0.40 \mathrm{~s}$ per frame. Each Astronomical Observation Request (AOR) lasted 6.5 hr, including 30 minutes overhead for the Pointing Calibration and Reference Sensor peak-up sequence. This step allowed GJ 3470 to be precisely

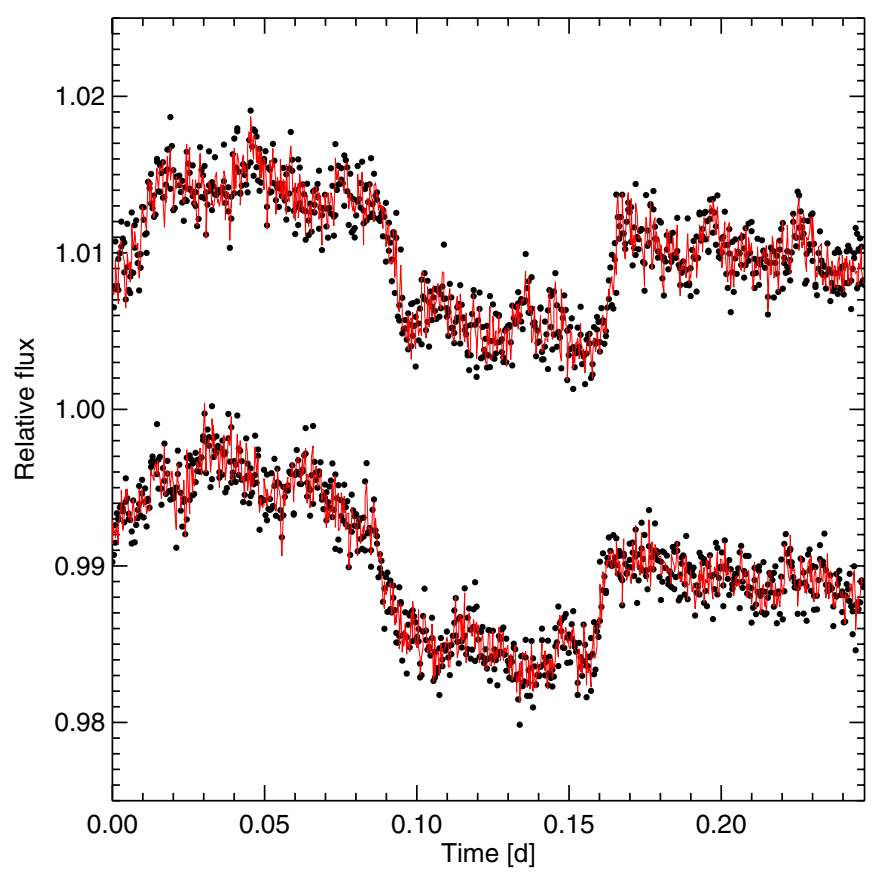

Figure 1. Spitzer/IRAC $4.5 \mu \mathrm{m}$ photometry. Raw photometry from the two AORs is displayed after normalization. The optimal baseline model (logarithmic ramp model added to a second-order polynomial fit for the centroid position and a time-dependent trend (see Section 3) is superimposed for each AOR in red. (A color version of this figure is available in the online journal.)

slewed on the position of maximum sensitivity on the $4.5 \mu \mathrm{m}$ channel subarray field-of-view (Ingalls et al. 2012; Demory et al. 2012b). All data were processed by the Spitzer pipeline version S19.1.0, which produced the basic calibrated data necessary for our reductions. We first convert fluxes from the Spitzer units of specific intensity (MJy/sr) to photon counts, and transform the data timestamps from BJD $\mathrm{UTC}_{\mathrm{TC}}$ to $\mathrm{BJD}_{\mathrm{TDB}}$ following Eastman et al. (2010). We then perform aperture photometry on each subarray image using the APER routine from the IDL Astronomy User's Library. ${ }^{14}$ We compute the stellar fluxes in aperture radii ranging between 1.8 and 4.0 pixels, the best results being obtained with an aperture radius of 3 pixels. We use background annuli extending from 11 to 15.5 pixels from the Point Response Function center. For each block of 64 subarray images, we discard the discrepant values for the measurements of flux, background, and $x-y$ centroid positions using a $10 \sigma$ median clipping for the four parameters. We then average the resulting values, the photometric errors being taken as the uncertainties on the average flux measurements. At this stage, a $50 \sigma$ clipping moving average is used on the resulting light curve to discard obviously discrepant subarrayaveraged fluxes. Close examination of the resulting time-series reveals a sharp increase of the background and stellar fluxes, corresponding to the well-known "ramp" effect seen in other warm Spitzer observations (see, e.g., Knutson et al. 2012). The raw photometry for both AORs is shown in Figure 1.

\subsection{WIYN Speckle Observations}

We supplemented our GJ 3470 b Spitzer photometry with speckle observations to explore the possibility of blended companions at close angular separations from GJ 3470. Speckle observations of GJ 3470 were obtained at the WIYN $3.5 \mathrm{~m}$

\footnotetext{
$\overline{14 \text { http://idlastro.gsfc.nasa.gov/contents.html }}$
} 


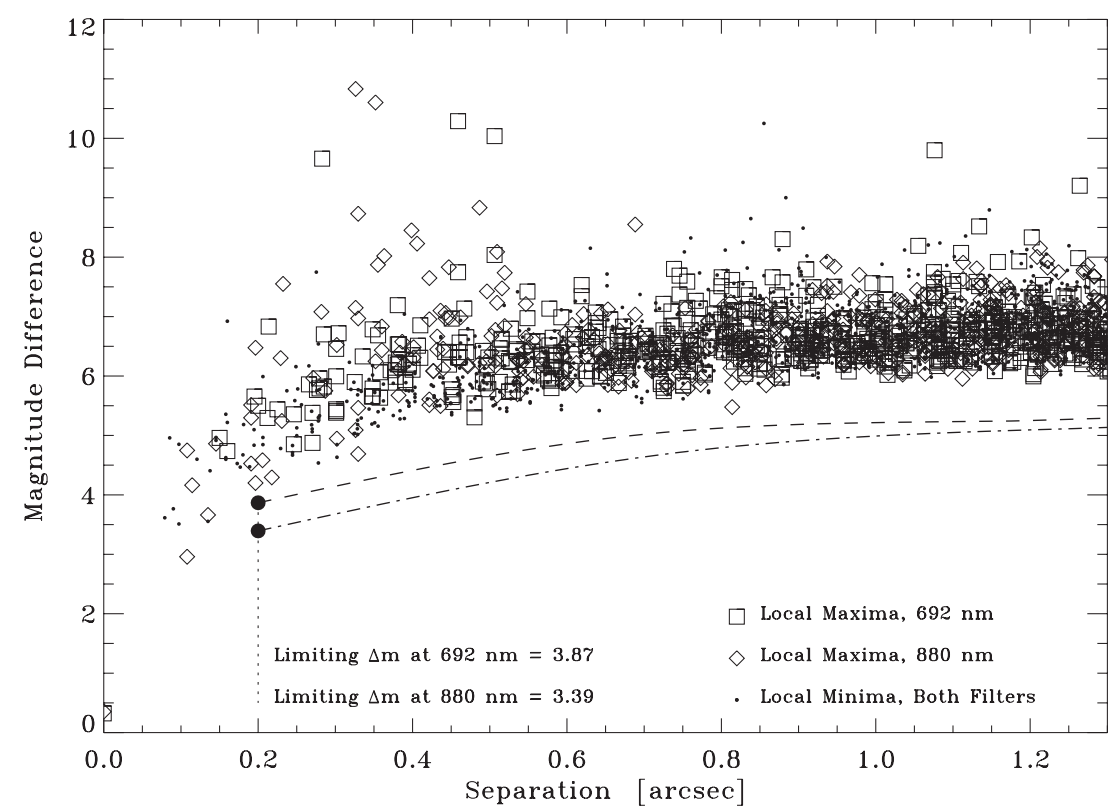

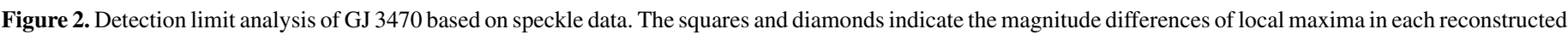

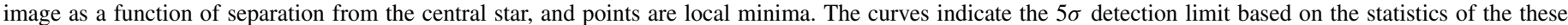

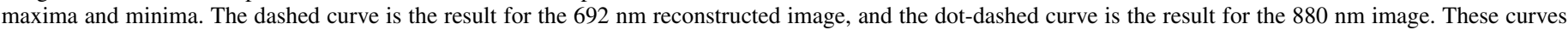
indicate a greater than 4 mag sensitivity in the limiting $\Delta m$ at most separations.

telescope on 2012 December 2. The camera used was the Differential Speckle Survey Instrument, which is described by Horch et al. (2009). It is a dual-channel instrument that records images in two colors simultaneously. In the case of this observation, the filters used had center wavelengths of $692 \mathrm{~nm}$ and $880 \mathrm{~nm}$, with filter widths of 40 and $50 \mathrm{~nm}$, respectively. A speckle sequence of $300050 \mathrm{~ms}$ frames was taken on the target, followed by 1000 frames taken on a bright point source (HR 3163) located near in the sky to GJ 3470. These latter data are used as an estimate of the speckle transfer function for deconvolution in the reduction process. Reconstructed images are formed from the speckle data using the technique of bispectral analysis, which is described, e.g., by Horch et al. (2012). We then analyze the final images to determine the detection limits of faint companions near GJ 3470 using the technique described in the same paper.

Figure 2 shows these detection limits based on the final diffraction-limited images in each filter. It is clear that there is no companion to the limit of our detection capabilities at a separation greater than 0.2 . At 0.2 the limiting $\Delta m$ for the $692 \mathrm{~nm}$ image is $3.87 \mathrm{mag}$, and for the $880 \mathrm{~nm}$ image it is $3.39 \mathrm{mag}$. Inside of this limit, as one approaches the central star, the limiting $\Delta m$ becomes smaller as the peaks and valleys of the reconstructed image get larger. In studying the two images, we find that none of the peaks near the central star are in the same position in both images, which is a good indication that they are probably not real stars but noise peaks. One of the advantages of the two independent channels in the instrument is to see if the positions of faint peaks match. We conclude that, to the limit of our detection at WIYN, there is no resolvable companion.

\subsection{Magellan/FIRE Near-infrared Spectroscopy}

Spectroscopic properties such as the effective temperature, $T_{\text {eff }}$, and metallicity, $[\mathrm{Fe} / \mathrm{H}]$, needed to establish the physical parameters of the parent stars of transiting planets have usually been difficult to determine for M dwarfs. Several studies in the past year have presented calibrations of $[\mathrm{Fe} / \mathrm{H}]$ or $T_{\text {eff }}$ in terms of easily measurable spectroscopic indices in the $H$-band and $K$-band regions that represent a significant advancement in the field. Toward this goal, we obtained a near-infrared spectrum of GJ 3470 on 2012 November 12 with the Folded-port Infrared Echellette (FIRE) spectrograph at the $6.5 \mathrm{~m}$ Magellan Baade telescope. FIRE delivers $R=6000$ spectra from 0.83 to $2.5 \mu \mathrm{m}$ in a single-object, cross-dispersed setup (Simcoe et al. 2008). We used an exposure time of 8.5 minutes on GJ 3470 and $105 \mathrm{~s}$ on the A0V telluric standard HD 58296. We reduce the data using FIRE's pipeline FIREHOSE, which employs the methods of Vacca et al. (2003) for telluric correction. An internal ThAr lamp provides wavelength calibration of both the GJ 3470 and HD 58296 spectra. The signal-to-noise ratio in the reduced spectrum is $>200$ in the $K$ band, where $\mathrm{H}_{2} \mathrm{O}$ features near 2.21 and $2.26 \mu \mathrm{m}$ fall on order 20 of FIRE's 21 cross-dispersed grating orders. Strong $\mathrm{OH}$ emission lines from the sky (which can introduce shot noise and residuals from sky subtraction) do not fall directly on these features. The measurement of various spectroscopic indices from this FIRE observation is described in Section 3.2.1.

\section{DATA ANALYSIS}

\subsection{Spitzer Photometry}

\subsubsection{Baseline Model Selection}

We first perform an individual analysis of each Spitzer AOR to determine the optimal baseline model, which accounts for time- and position-dependent systematic effects relevant to our IRAC $4.5 \mu \mathrm{m}$ observations. We employ for this purpose our adaptive Markov Chain Monte Carlo (MCMC) implementation described by Gillon et al. (2010). We test six baseline models of increasing complexity, and compare their Bayesian information criteria (BIC; see, e.g., Gelman et al. 2003) to choose the baseline model that yields the highest marginal likelihood. We correct for the well-known "pixel-phase" effect using a second- to fourth-order $x-y$ position-dependent polynomial, 
while the "ramp" is corrected using a second-order logarithmic model. We also check for time-dependent trends of instrumental and/or stellar origin by adding linear or quadratic functions of time to our baseline models. We additionally explore the correlation of the stellar flux and background time series with the full width at half-maximum of the point response function (Demory et al. 2012b). We find for both AORs the lowest BIC to correspond to a model including a second-order positiondependent polynomial, a second-order logarithmic ramp, and a time-dependent linear trend. Our analysis yields an rms of $362 \mathrm{ppm}$ and $369 \mathrm{ppm}$ per 5 minute interval in the first and second AORs, respectively, with negligible contribution from correlated noise.

\subsubsection{Determination of the Stellar Density}

We perform a combined MCMC fit including our two Spitzer transits and the 61 HARPS radial velocities (RVs) published in the discovery paper (Bonfils et al. 2012). The main goal of this step is to derive the stellar density from the Spitzer photometry (Seager \& Mallén-Ornelas 2003), to enable the derivation of the stellar and planetary physical parameters. The following system parameters ("jump parameters") are left free in the MCMC fit, using uniform priors: the orbital period $P$, transit depth $d F$ (planet-to-star area ratio, $\left.\left(R_{p} / R_{\star}\right)^{2}\right)$, transit duration $W$, time of minimum light $T_{0}$, impact parameter $b=a \cos i / R_{\star}$, the parameter $K^{\prime}=K \sqrt{1-e^{2}} P^{1 / 3}$, where $K$ is the RV semiamplitude, $\sqrt{e} \cos \omega$ and $\sqrt{e} \sin \omega$. We use a quadratic law for the limb-darkening. We draw the theoretical values and corresponding uncertainties of the coefficients $u_{1}$ and $u_{2}$ from the tables of Claret \& Bloemen (2011) for the $T_{\text {eff }}, \log g$, and $[\mathrm{Fe} / \mathrm{H}]$ determinations reported in Section 4. We use the resulting distributions for $u_{1}$ and $u_{2}$ as normal priors in our MCMC fit. We use the linear combinations $c_{1}=2 u_{1}+u_{2}$ and $c_{2}=u_{1}-2 u_{2}$ as jump parameters, rather than $u_{1}$ and $u_{2}$, to minimize the correlations of the resulting uncertainties (Holman et al. 2006). At each step of the MCMC fit, the stellar density is derived from this set of parameters and Kepler's third law. We run two chains of $10^{5}$ steps each, where the first $20 \%$ are discarded. We assess the good convergence and mixing of the chains employing the Gelman-Rubin statistic (Gelman \& Rubin 1992). We add a $2.0 \mathrm{~m} \mathrm{~s}^{-1}$ jitter contribution in quadrature to the RV error bars to match the rms of the residuals. This first combined run yields an eccentricity signal compatible with a circular orbit $(\sqrt{e} \cos \omega=-0.09 \pm 0.14$ and $\sqrt{e} \sin \omega=0.00 \pm 0.22$ ). We therefore repeat the fit setting $\sqrt{e} \cos \omega$ and $\sqrt{e} \sin \omega$ to zero. The difference in BIC between an eccentric and a circular orbit is $\triangle \mathrm{BIC}=15$, translating to an odds ratio of $\sim 1800$, hence favoring the circular model we adopt in the following. Our analysis assuming a circular orbit yields a stellar density $\rho_{\star}=2.91_{-0.33}^{+0.37} \rho_{\odot}$ that we use as a constraint for the derivation of the stellar parameters in Section 4. The phase-folded Spitzer light curve is shown in Figure 3.

\subsection{Spectroscopic Measurements}

\subsubsection{FIRE Spectral Analysis}

We measure a number of spectral features in our FIRE spectrum of GJ 3470 for the purpose of deriving its spectroscopic properties, particularly $[\mathrm{Fe} / \mathrm{H}]$ and $T_{\text {eff }}$, using recent calibrations presented for $\mathrm{M}$ dwarfs. We follow closely the prescriptions of Rojas-Ayala et al. (2012), Terrien et al. (2012), and Mann et al. (2013) for measuring equivalent widths (EWs) as well as water

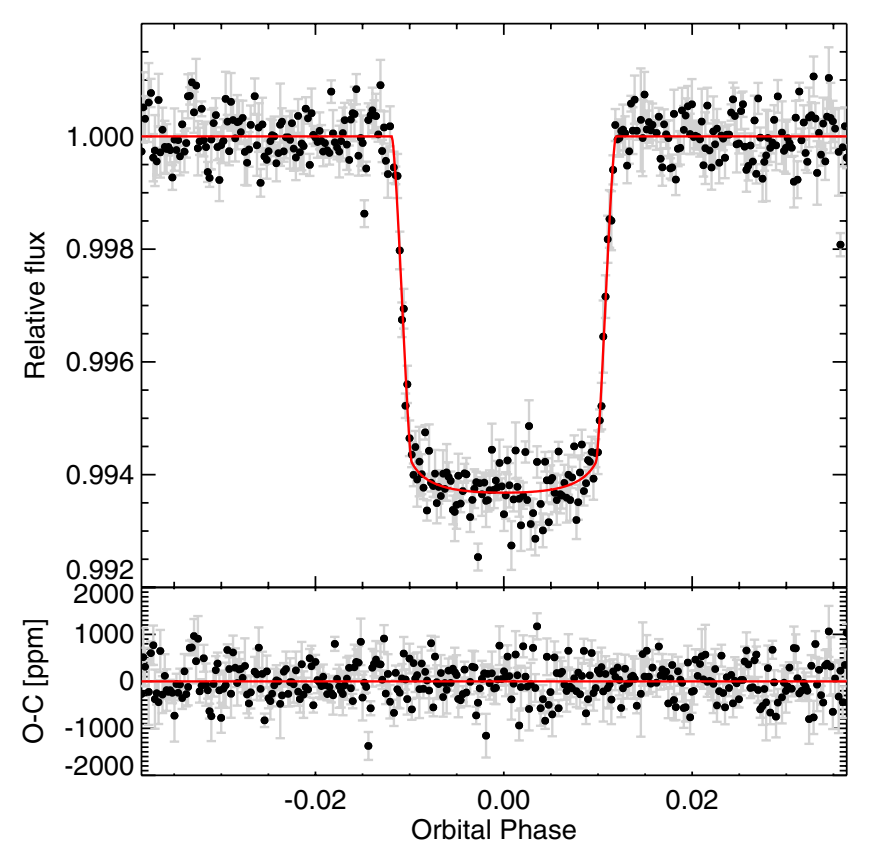

Figure 3. GJ $3470 \mathrm{~b}$ detrended and phase-folded transit light curve combining our two $4.5 \mu \mathrm{m}$ Spitzer/IRAC visits, with the best-fit transit model superimposed (see Section 5). Data points are binned in 2 minute intervals, and residuals are shown in the bottom panel.

(A color version of this figure is available in the online journal.)

indices, and to estimate the pseudo-continuum needed for the above metallicity calibrations.

For the Rojas-Ayala et al. (2012) calibration, we measure the Na doublet $(2.206$ and $2.209 \mu \mathrm{m})$ and the Ca triplet $(2.261$, 2.263, and $2.265 \mu \mathrm{m}$ ) following the integration limits and continuum points of their Table 2 . The pseudo-continuum flux of each feature is taken from a linear fit to the median flux within a $3 \mathrm{~nm}$ region around each continuum point. The water index, $\mathrm{H}_{2} \mathrm{O}-\mathrm{K} 2$, is measured following Equation (5) of Rojas-Ayala et al. (2012). For the application of the $H$-band and $K$-band metallicity calibrations of Terrien et al. (2012), we measure the EW of the $\mathrm{Na}(2.2074 \mu \mathrm{m}), \mathrm{Ca}(1.6159,1.6203,2.2638 \mu \mathrm{m})$, and $\mathrm{K}(1.5171 \mu \mathrm{m})$ features following the prescription detailed in Section 3.1 of their study. The pseudo-continuum is estimated by fitting a fourth-order Legendre polynomial to the regions shown in their Figure 1(A) for the $H$ band, and in their Figure 1(B) for the $K$ band. The water indices, $\mathrm{H}_{2} \mathrm{O}-\mathrm{H}$ and $\mathrm{H}_{2} \mathrm{O}-\mathrm{K}$, are also measured following the definitions in their paper. For the Mann et al. (2013) calibration, the EW of the metal-sensitive features F19 $(2.2079 \mu \mathrm{m})$, F20 $(2.3242 \mu \mathrm{m})$, and F22 $(2.3844 \mu \mathrm{m})$ in the $K$ band are measured using the parameters listed in their Table 5 . The pseudo-continuum is measured by a linear fit in the spectral regions specified in their Table 4, immediately redward and blueward of each feature. The water index used for this $K$-band calibration is the same as the one described by Rojas-Ayala et al. (2012).

The EWs and spectral indices computed from our FIRE spectrum as described above yield the metallicities for GJ 3470 shown in the first four lines of Table 1. A spectroscopic estimate of the effective temperature of GJ 3470 is obtained using the temperature-sensitive $\mathrm{H}_{2} \mathrm{O}-\mathrm{K} 2$ index in the $K$ band as defined by Rojas-Ayala et al. (2012). The result is $3750 \pm 300 \mathrm{~K}$.

\subsubsection{HARPS Spectral Analysis}

An additional spectroscopic estimate of the metallicity of GJ 3470 is obtained from a recent calibration (V. Neves et al., in 
Table 1

Metallicity Estimates (dex) for GJ 3470 from Near-infrared and Visible Spectroscopy

\begin{tabular}{lcc}
\hline \hline Calibration Reference & Bandpass & Value \\
\hline Rojas-Ayala et al. (2012) & $K$ & $+0.15 \pm 0.17$ \\
Terrien et al. (2012) & $H$ & $+0.25 \pm 0.12$ \\
Terrien et al. (2012) & $K$ & $+0.19 \pm 0.12$ \\
Mann et al. (2013) & $K$ & $+0.32 \pm 0.11$ \\
Neves et al. (2013) & Visible & $+0.08 \pm 0.10$ \\
Adopted & $\ldots$ & $+0.20 \pm 0.10$ \\
\hline
\end{tabular}

preparation) based on the visible-light HARPS spectra of Bonfils et al. (2012). This calibration was established on the basis of EWs measured for a total of 4441 lines in the spectra of 55 stars from the HARPS volume-limited M-dwarf sample (Bonfils et al. 2013), and is anchored on existing photometric calibrations for metallicity (Neves et al. 2012) and effective temperature (Casagrande et al. 2008). The procedure, described briefly in the appendix of the study by Neves et al. (2013), achieves an improved precision over previous methods of 0.10 dex. The result of this measurement for GJ 3470 is $+0.08 \pm 0.10$, and is also collected in Table 1.

The five estimates of $[\mathrm{Fe} / \mathrm{H}]$ from the FIRE and HARPS spectra are consistent with each other, and we therefore adopt for the remainder of the paper the weighted average, $[\mathrm{Fe} / \mathrm{H}]=$ $+0.20 \pm 0.10$, in which the uncertainty is a more conservative estimate than the formal error of the mean.

\section{STELLAR CHARACTERIZATION}

Mass $\left(M_{\star}\right)$ and radius $\left(R_{\star}\right)$ estimates for exoplanet host stars are typically obtained by appealing to stellar evolution models. For M dwarfs this, too, has been problematic (beyond the challenges for determining $T_{\text {eff }}$ and $[\mathrm{Fe} / \mathrm{H}]$ alluded to earlier in Section 2.3) because of known disagreements between predictions from theory and accurate measurements of $M_{\star}$ and $R_{\star}$ for low-mass stars in double-lined eclipsing binaries (see, e.g., Torres 2013 and references therein). We therefore rely here exclusively on empirical relations, on the mean stellar density inferred from our Spitzer light curve in Section 3.1.2 $\left(\rho_{\star}=2.91_{-0.33}^{+0.37} \rho_{\odot}\right)$, and on brightness measurements for GJ 3470 from Two Micron All Sky Survey (2MASS) and in the optical ( $V=12.33 \pm 0.01$; Weis 1986; Evans et al. 2002; Zacharias et al. 2013). For a given parallax and ignoring extinction, the near-infrared mass-luminosity $(M-L)$ relations of Delfosse et al. (2000) provide estimates of the absolute mass, and are insensitive to metallicity. On the other hand, the surface-brightness (SB) relations by Kervella et al. (2004) allow one to estimate the angular diameter, which may be converted to a linear radius with knowledge of the parallax. The latter relations are valid for $[\mathrm{Fe} / \mathrm{H}]$ between -0.5 and +0.5 , and are thus applicable to GJ 3470 , with its metallicity of $[\mathrm{Fe} / \mathrm{H}]=+0.20 \pm 0.10$. While a trigonometric parallax has not been measured for this star, we may use the above relations simultaneously to solve for the distance that yields values of $M_{\star}$ and $R_{\star}$ consistent with the measured mean density.

We proceeded in a Monte Carlo fashion, drawing all measured quantities ( $V J H K_{s}$ photometry and Spitzer mean density) from appropriate Gaussian distributions. For each set of draws we solve for the value of the parallax that gives a mass and radius through the $M-L$ and SB relations resulting in a mean density equal to the randomly drawn value of $\rho_{\star}$ for the set. We repeat the process $10^{5}$ times, and adopt as final values the mode of
Table 2

Adopted System Parameters for GJ 3470 from our MCMC Fit of Section 5

\begin{tabular}{|c|c|}
\hline Parameter & Value \\
\hline \multicolumn{2}{|c|}{ Jump parameters } \\
\hline Planet/star area ratio $R_{p} / R_{s}$ & $0.07798_{-0.00045}^{+0.00046}$ \\
\hline$b=a \cos i / R_{\star}\left(R_{\star}\right)$ & $0.40_{-0.08}^{+0.06}$ \\
\hline Transit width $W(\mathrm{~d})$ & $0.0791 \pm 0.0005$ \\
\hline$T_{0}-2,450,000\left(\mathrm{BJD}_{\mathrm{TDB}}\right)$ & $6090.47701 \pm 0.00010$ \\
\hline Orbital period $P(\mathrm{~d})^{\mathrm{a}}$ & $3.33665 \pm 0.00005$ \\
\hline $\mathrm{RV} K^{\prime}\left(\mathrm{m} \mathrm{s}^{-1} \mathrm{~d}^{1 / 3}\right)$ & $13.4 \pm 1.2$ \\
\hline$\sqrt{e} \cos \omega$ & 0.0 (fixed) \\
\hline$\sqrt{e} \sin \omega$ & 0.0 (fixed) \\
\hline$c_{1}=2 u_{1}+u_{2}$ & $0.246 \pm 0.027$ \\
\hline$c_{2}=u_{1}-2 u_{2}$ & $-0.329 \pm 0.020$ \\
\hline \multicolumn{2}{|c|}{ Stellar parameters } \\
\hline$u_{1}$ & $0.033 \pm 0.015$ \\
\hline$u_{2}$ & $0.181 \pm 0.010$ \\
\hline Mean density $\rho_{\star}\left(\rho_{\odot}\right)$ & $2.91_{-0.33}^{+0.37}$ \\
\hline Surface gravity $\log g_{\star}(\mathrm{cgs})$ & $4.658 \pm 0.035$ \\
\hline Mass $M_{\star}\left(M_{\odot}\right)^{\mathrm{b}}$ & $0.539_{-0.043}^{+0.047}$ \\
\hline Radius $R_{\star}\left(R_{\odot}\right)^{\mathrm{b}}$ & $0.568_{-0.031}^{+0.037}$ \\
\hline Parallax $\pi$ (mas) $^{\mathrm{b}}$ & $32.4_{-1.9}^{+2.1}$ \\
\hline Distance $(\mathrm{pc})^{\mathrm{b}}$ & $30.7_{-1.7}^{+2.1}$ \\
\hline Effective temperature $T_{\text {eff }}(\mathrm{K})^{\mathrm{b}}$ & $3600 \pm 100$ \\
\hline Metallicity $[\mathrm{Fe} / \mathrm{H}](\mathrm{dex})^{\mathrm{b}}$ & $+0.20 \pm 0.10$ \\
\hline \multicolumn{2}{|c|}{ Planetary parameters } \\
\hline RV semi-amplitude $K\left(\mathrm{~m} \mathrm{~s}^{-1}\right)$ & $8.9 \pm 1.1$ \\
\hline Orbital semi-major axis $a(\mathrm{AU})$ & $0.03557_{-0.00100}^{+0.00096}$ \\
\hline Orbital inclination $i(\mathrm{deg})$ & $88.3_{-0.4}^{+0.5}$ \\
\hline Mean density $\rho_{p}\left(\mathrm{~g} \mathrm{~cm}^{-3}\right)$ & $0.72_{-0.12}^{+0.13}$ \\
\hline Surface gravity $\log g_{p}(\mathrm{cgs})$ & $2.76_{-0.07}^{+0.06}$ \\
\hline Mass $M_{p}\left(M_{\oplus}\right)$ & $13.9_{-1.4}^{+1.5}$ \\
\hline Radius $R_{p}\left(R_{\oplus}\right)$ & $4.83_{-0.21}^{+0.22}$ \\
\hline \multicolumn{2}{|c|}{ Individual transit timings } \\
\hline$T_{0,1}-2,450,000\left(\mathrm{BJD}_{\mathrm{TDB}}\right)$ & $6090.47705 \pm 0.00014$ \\
\hline$T_{0,2}-2,450,000\left(\mathrm{BJD}_{\mathrm{TDB}}\right)$ & $6093.81372 \pm 0.00015$ \\
\hline
\end{tabular}

Notes

a Derived using our two Spitzer light curves along with published ground based photometry and RVs (see Section 5).

b Parameters derived either in Section 3 or in Section 4, and repeated here for convenience.

the corresponding posterior probability distributions, assigning $1 \sigma$ uncertainties given by the 15.85 and 84.13 percentiles of those distributions. We obtain $M_{\star}=0.539_{-0.043}^{+0.047} M_{\odot}$ and $R_{\star}=0.568_{-0.031}^{+0.037} R_{\odot}$, and a parallax of $\pi=32.4_{-1.9}^{+2.1}$ mas, corresponding to a distance of $30.7_{-1.7}^{+2.1} \mathrm{pc}$. The mass is an average of the $\mathrm{J}-, \mathrm{H}$-, and $\mathrm{K}$-band relations by Delfosse et al. (2000), each of which is assumed conservatively to carry an uncertainty of $10 \%$. The radius is an average of the two SB relations of Kervella et al. (2004) that yield the smallest scatter in the angular diameters (about $1 \%$ for the relations that depend on $V-H$ and $V-K$ ). Prior to using them, the 2MASS magnitudes are converted to the native photometric system of the $M-L$ and SB relations (CIT and Johnson, respectively) using the transformations of Carpenter (2001). The uncertainties listed above include all photometric errors, the error in $\rho_{\star}$, as well as the scatter of the empirical relations. We note that our stellar mass is very close to that reported by Bonfils et al. (2012), but our radius is $13 \%$ larger. 


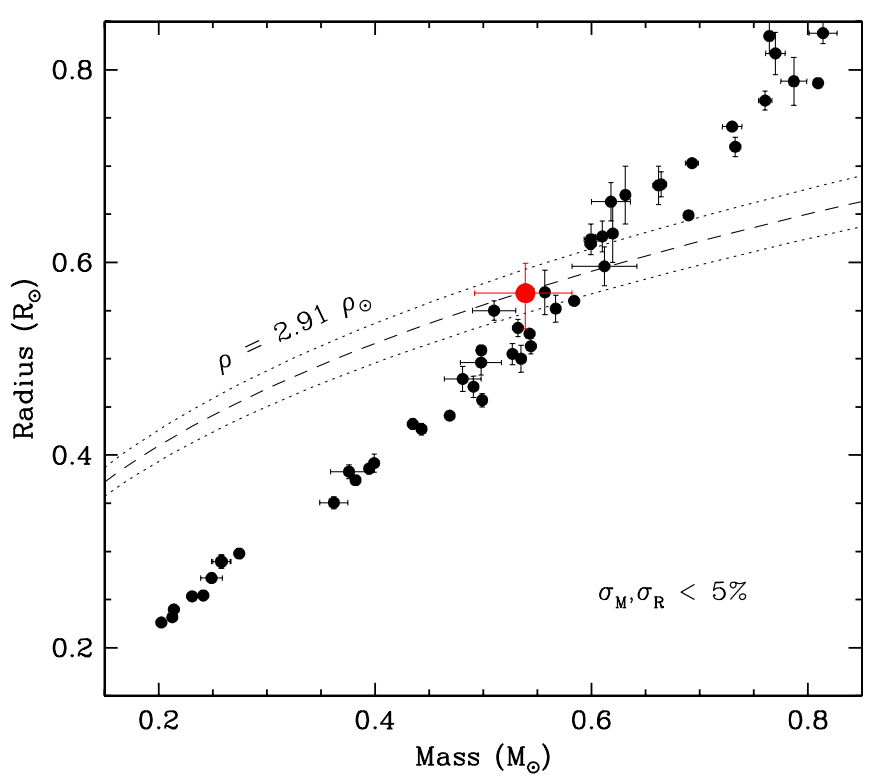

Figure 4. Mass and radius estimates for GJ 3470 (large red dot) compared with measurements for other low-mass stars in double-lined eclipsing binaries with relative errors in $M_{\star}$ and $R_{\star}$ less than 5\% (see Torres 2013). The constraint on the mean stellar density derived from our Spitzer observations is indicated with the dashed line (dotted lines representing the $1 \sigma$ uncertainties).

(A color version of this figure is available in the online journal.)

As a check on the above absolute radius determination, we obtain additional estimates of $R_{\star}$ from color indices and the calibrations recently published by Boyajian et al. (2012), which are based on angular diameter measurements from the CHARA interferometer and HIPPARCOS parallaxes, and have a dependence on metallicity. Results using $V-J, V-H$, and $V-K_{s}$ for the measured metallicity of GJ 3470 give very consistent values for $R_{\star}$ averaging $0.513 \pm 0.043 R_{\odot}$, in agreement with our Spitzer-based determination within about $1 \sigma$. Figure 4 displays the location of GJ 3470 in the mass-radius diagram for low-mass stars, along with the measurements for all other such objects in double-lined eclipsing binaries that have relative measurement precisions under $5 \%$ for $M_{\star}$ and $R_{\star}$. The constraint afforded by the mean stellar density is also indicated.

While an estimate of the effective temperature of the star was obtained earlier using our FIRE spectrum, the precision is relatively low. We obtain a further estimate using the color/temperature calibrations of Boyajian et al. (2012), which are based on bolometric fluxes and angular diameter measurements, and include metallicity terms. The $V-J, V-H$, and $V-K_{s}$ indices along with our adopted value of $[\mathrm{Fe} / \mathrm{H}]$ lead to a weighted average temperature of $3630 \pm 100 \mathrm{~K}$. A final $T_{\text {eff }}$ estimate is inferred from the same three indices and the color/ temperature calibrations of Casagrande et al. (2008), which rely on the Infrared Flux Method. However, these relations do not take into account the metallicity, and implicitly assume a composition near solar whereas GJ 3470 is metal-rich. Therefore, the resulting estimate $(3360 \pm 100 \mathrm{~K})$ requires an adjustment for metallicity. We determine this by using the stellar evolution models of Dotter et al. (2008) in a differential sense, first reading off from a $[\mathrm{Fe} / \mathrm{H}]=+0.20$ isochrone the stellar mass that yields the same color indices as we measure, and then comparing the corresponding temperature with that for a star of the same mass on a solar-metallicity isochrone. This exercise is insensitive to the age adopted for the isochrone. Consistent results using the three color indices separately give an average correction of $+140 \mathrm{~K}$, which results in a final temperature of $3500 \pm 150 \mathrm{~K}$. As the two photometric determinations above are consistent with each other and with the spectroscopic determination in Section 3.2.1, we adopt the weighted average of the three values, $T_{\text {eff }}=3600 \pm 100 \mathrm{~K}$.

While this paper was under review, we learned that Pineda et al. (2013) performed an independent characterization of GJ 3470's stellar properties. We refer the reader to that study for a description of their analysis and results.

\section{PLANETARY AND ORBITAL PARAMETERS}

Our final parameters for GJ $3470 \mathrm{~b}$ were derived using the stellar properties from the preceding section, and an MCMC fit analogous to that described in Section 3 with the addition of a prior on the stellar mass. For this prior we used the posterior probability distribution derived in Section 4, drawing from it a random value of the mass at each step of the MCMC fit. As before, we included the light curves from both Spitzer visits, along with the 61 HARPS RVs reported by Bonfils et al. (2012). The results are presented in Table 2, where the value adopted for each parameter is the median of the corresponding marginalized posterior distribution from the MCMC fit. Error bars are the corresponding $68.3 \%$ probability intervals from the same distributions. The final model and phase-folded Spitzer light curves are displayed in Figure 3.

We find for GJ $3470 \mathrm{~b}$ a radius of $R_{p}=4.83_{-0.21}^{+0.22} R_{\oplus}$, which is $13 \%$ larger than previously reported in the literature. This increase is driven mainly by the larger stellar radius from Section 4. Combining the planetary radius with the mass $M_{p}=13.9_{-1.4}^{+1.5} M_{\oplus}$ that relies on the RV data set yields a very low planetary density of $\rho_{p}=0.72_{-0.12}^{+0.13} \mathrm{~g} \mathrm{~cm}^{-3}$, which is $33 \%$ smaller than the estimate in the discovery paper. These planetary parameters are also listed in Table 2.

Finally, we performed a new fit for the purpose of assessing the robustness of the orbital period determination for GJ $3470 \mathrm{~b}$, which in our solution is constrained both by the two Spitzer transits and the RVs. However, the two Spitzer visits are consecutive (2012 June 11 and 15), so the lever arm for the orbital period determination is very short. We therefore incorporated the two TRAPPIST transit light curves from Bonfils et al. (2012), as well as the ones from EulerCam and the NITES telescope. The light curves from the first two sources show only the ingress portion of the transit, but may still be combined with our two full Spitzer light curves that constrain the transit shape, if we assume the latter does not change across wavelengths. The NITES light curve has a higher level of correlated noise, but does cover the transit completely. These ground-based light curves were obtained between 2012 February and April, and therefore contribute to build up a much longer baseline.

As expected, most of the system parameters in this new fit are tightly constrained by the Spitzer photometry alone, but the period is considerably improved. The new value is included in Table 2, and is only $19 \pm 11 \mathrm{~s}$ shorter than the one that relies on the two Spitzer transits alone.

\section{INTERIOR COMPOSITION OF A LOW-DENSITY EXO-NEPTUNE}

GJ 3470 b presents a valuable test case for planet formation and evolution theories. It stands out from the crowd of accumulating transiting exo-Neptunes due to its low mean density and bright $\mathrm{M}$ dwarf host star. GJ 3470 b's measured radius is $20 \% \pm 6 \%$ larger than Uranus $\left(R_{\uparrow}=4.01 R_{\oplus}\right)$ despite having a 


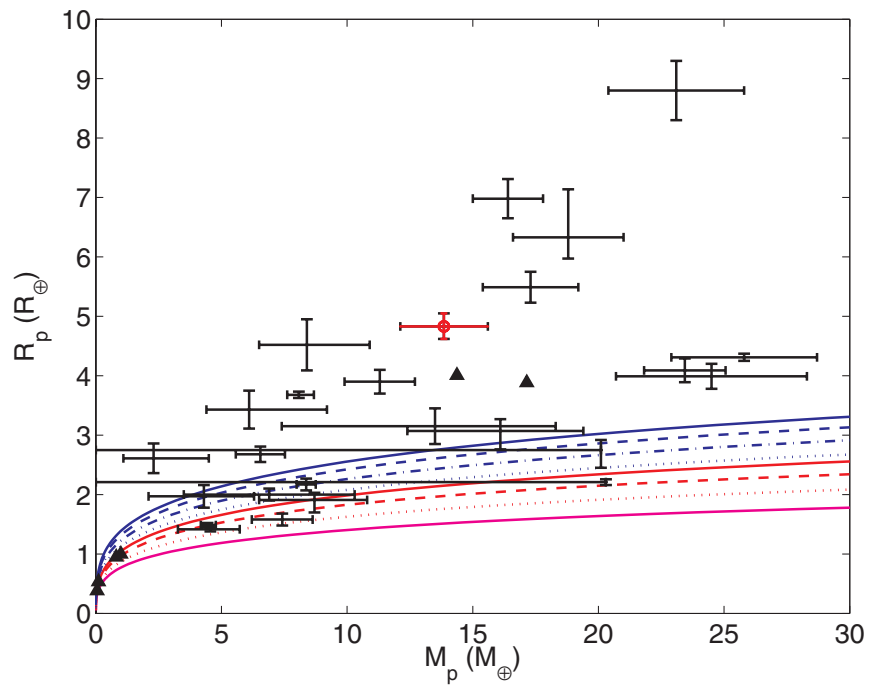

Figure 5. Mass-radius relationships of small transiting planets. GJ $3470 \mathrm{~b}$ is highlighted in red. Other small transiting exoplanets with dynamical mass constraints (CoRoT-7 b, Kepler-4 b, Kepler-10 b, Kepler-11 b, c, d, e, f, Kepler18 b, c, d, Kepler-19 b, Kepler-20 b, c, d, Kepler-30 b, d, Kepler-36 b, c, 55 Cnc e, GJ 1214 b, GJ 436 b, HAT-P-11 b, and HAT-P-26 b) are plotted in black. The solar system planets are indicated with solid triangles. The curves are illustrative constant-temperature mass-radius relations from Seager et al. (2007). The solid lines are homogeneous-composition planets: water ice (blue solid), $\mathrm{MgSiO}_{3}$ perovskite (red solid), and iron (magenta solid). The non-solid lines are mass-radius relations for differentiated planets: $75 \%$ water ice, $22 \%$ silicate shell, and 3\% iron core (blue dashed); Ganymede-like with $45 \%$ water ice, $48.5 \%$ silicate shell, and $6.5 \%$ iron core (blue dot-dashed); $25 \%$ water ice, $52.5 \%$ silicate shell, and $22.5 \%$ iron core (blue dotted); Earth-like with $67.5 \%$ silicate mantle and $32.5 \%$ iron core (red dashed); and Mercury-like with $30 \%$ silicate mantle and $70 \%$ iron core.

(A color version of this figure is available in the online journal.)

similar mass $\left(M_{\hat{\wp}}=14.5 M_{\oplus}\right)$. The planet radius corresponds to roughly $20 \%$ of its Roche lobe radius. Among currently known low-mass $\left(M_{p}<30 M_{\oplus}\right)$ transiting planets, only the Kepler-11, Kepler-18, Kepler-30, and HAT-P-26 systems have planets with lower densities (see Figure 5).

GJ 3470 b must have acquired $\mathrm{H} / \mathrm{He}$ gas from the protoplanetary nebula. Alternative gas layer sources such as sublimated ices and outgassing from a rocky interior may be important for less massive, more dense planets (such as GJ 1214b; Rogers $\&$ Seager 2010b), but cannot be the dominant gas layer source for GJ 3470 b. Its bulk density is too low for astrophysical ices $\left(\mathrm{H}_{2} \mathrm{O}, \mathrm{CO}_{2}\right.$, etc. $)$ alone to comprise the planet volatiles; significant quantities of light gases (hydrogen and helium) must be present. Further, GJ 3470 b's gas layer is too voluminous to have been formed by outgassing of light gases during formation; the planet radius exceeds the upper limit for outgassed planets from Rogers et al. (2011).

Nebular $\mathrm{H} / \mathrm{He}$ contributes between $5 \%$ and $24 \%$ to GJ 3470 b's mass, according to our interior structure models. Following Rogers \& Seager (2010b), we apply a fully differentiated model for the planet's interior structure consisting of (from the center of the planet outward) an iron core, silicate layer, ice layer, and $\mathrm{H} / \mathrm{He}$ gas envelope to explore which bulk compositions are consistent with the measured mass and radius of GJ 3470 b. Both the planet's bond albedo $A$ (which scales the equilibrium temperature $\left.T_{\mathrm{eq}}=(1-A)^{1 / 4}(683 \pm 27) \mathrm{K}\right)$, and the planet's intrinsic luminosity $L_{p}$ (a proxy for the poorly constrained age of the planet) are unknown. We adopt fiducial values of $A=0.3$ and $L_{p} / M_{p}=10^{-10} \mathrm{~W} \mathrm{~kg}^{-1}$, while also exploring the ranges of $A=0-0.5$ and $L_{p} / M_{p}=10^{-10.5} \mathrm{~W} \mathrm{~kg}^{-1}$ to $10^{-9.5} \mathrm{~W} \mathrm{~kg}^{-1}$. Figure 6 presents the $\mathrm{H} / \mathrm{He}$ gas mass fraction $\left(M_{\mathrm{XY}} / M_{p}\right)$ in our models as a function of the Fe-silicate- $\mathrm{H}_{2} \mathrm{O}$ abundances of the heavy element interior (assuming the median values of the planet mass and radius, and our nominal planet energy budget parameters). Varying the planet mass and radius within their $1 \sigma$ bounds, and considering a range of plausible planet energy budgets affects the $\mathrm{H} / \mathrm{He}$ mass fractions by up to \pm 0.05 . For a rocky Earth-like heavy element interior composition ( $32 \% \mathrm{Fe}, 68 \%$ silicate, $\left.0 \% \mathrm{H}_{2} \mathrm{O}\right)$, GJ 3470 b's $\mathrm{H} / \mathrm{He}$ envelope mass is constrained to $M_{\mathrm{XY}} / M_{p}=0.16 \pm 0.05$, while for a denser iron-enhanced Mercury-like rocky interior $(70 \% \mathrm{Fe}$, $30 \%$ silicate, $\left.0 \% \mathrm{H}_{2} \mathrm{O}\right), M_{\mathrm{XY}} / M_{p}=0.17 \pm 0.05$. Less $\mathrm{H} / \mathrm{He}$ is needed if GJ $3470 \mathrm{~b}$ has an ice-rich interior composition; for instance, for a heavy element interior with $16 \% \mathrm{Fe}, 34 \%$ silicate, $50 \% \mathrm{H}_{2} \mathrm{O}, M_{\mathrm{XY}} / M_{p}=0.12_{-0.04}^{+0.05}$.

Which heavy element interior compositions are plausible for GJ 3470 b? The planet interior ice-to-rock ratio is not constrained by measurements of the planets mass and radius alone, so we look to planet formation theory for insights. If GJ $3470 \mathrm{~b}$ formed beyond the snow line and migrated inward to its current orbit, its heavy element interior would be icerich. If instead GJ $3470 \mathrm{~b}$ formed in situ (inside the snow line) its heavy element interior would be rock dominated with a lower proportion of ices. Theoretical predictions for how much ice is likely included in planets formed inside the snow line of $\mathrm{M}$ dwarfs are a topic of ongoing debate. Ogihara \& Ida (2008) proposed that migration of planetesimals from beyond the snow line could supply icy material to the inner regions of the protoplanetary disk. On the other hand, Lissauer (2007) and Kennedy et al. (2007) predict that planets and planetesimals formed within $1 \mathrm{AU}$ of $\mathrm{M}$ dwarfs are unlikely to have large volatile inventories when the effect of the M dwarfs' pre-main sequence luminosity evolution is taken into account. In Figure 6 we present interior bulk compositions for the full range of ice-to-rock ratios.

\section{SUMMARY}

Our $4.5 \mu \mathrm{m}$ Spitzer observations have enabled us to refine the planetary and system parameters of the Neptune-size planet GJ $3470 \mathrm{~b}$, improving its radius to $R_{p}=4.8 \pm 0.2 R_{\oplus}$, which is $13 \%$ larger than previously reported in the literature. As a result, the revised planetary density, $\rho_{p}=0.72 \pm 0.13 \mathrm{~g} \mathrm{~cm}^{-3}$, is $33 \%$ smaller than before. These changes come mostly from revisions of the stellar parameters (particularly $R_{\star}$ ), which have been frustratingly difficult to determine accurately in the past due to known discrepancies between observations and standard stellar evolution models for lower main-sequence stars. In this paper we have relied for this only on empirical $M-L$ and SB relations that have been widely employed in other contexts, and on the strong constraint on the mean stellar density provided by our Spitzer observations. In the process we have inferred an accurate distance for the star.

GJ 3470 b provides a valuable example of an extremely low-density planet, representative of a significant portion of the exoplanet candidates found by the Kepler mission to date. The brightness of the host star $\left(K_{s}=7.99\right)$ combined with its large planet-to-star radius ratio renders GJ 3470 b a promising candidate for future atmospheric characterization, which could provide clues on its formation pathway. Indeed, GJ 3470 b's low surface gravity translates to a large atmospheric scale height for a given atmospheric composition, favoring follow-up studies applying transmission spectroscopy. GJ 3470 b, GJ 436 b, and 


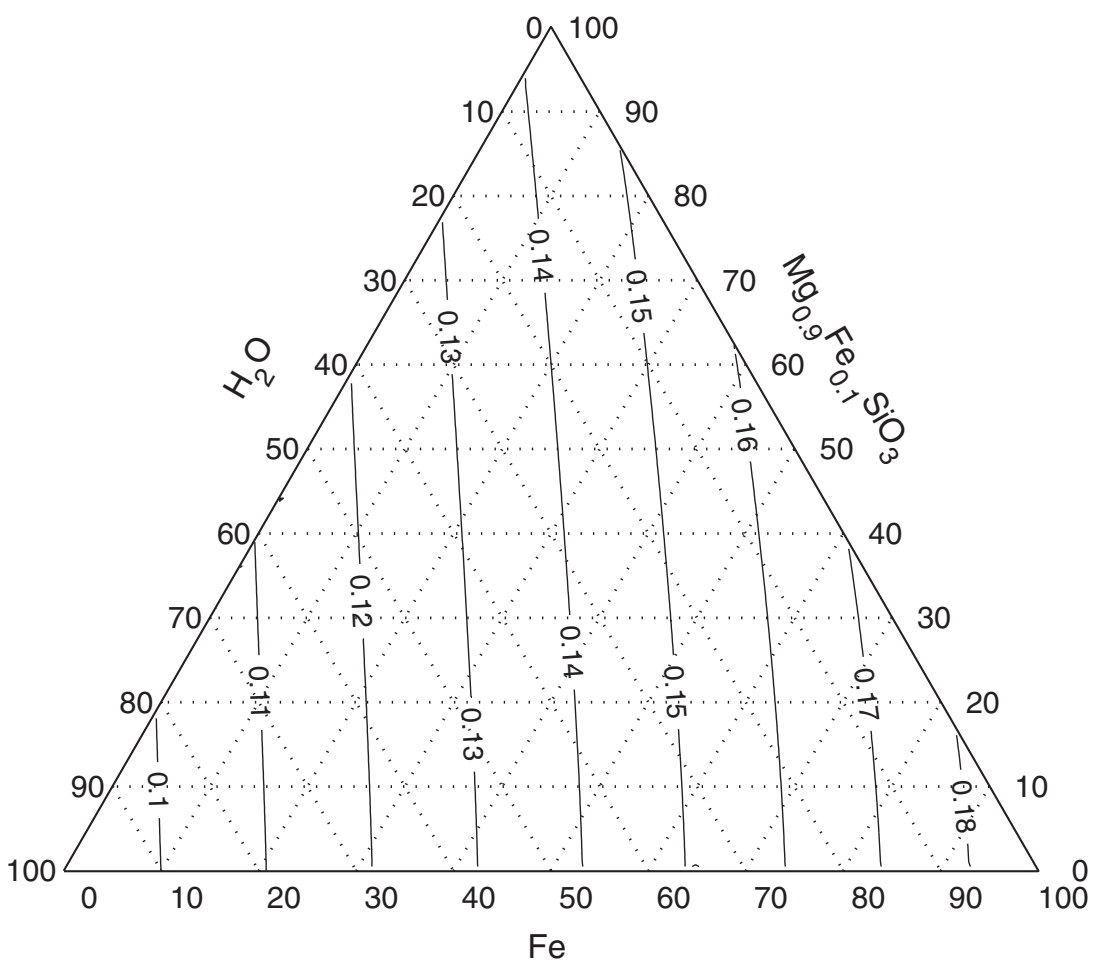

Figure 6. Fraction of GJ 3470 b's mass contributed by $\mathrm{H} / \mathrm{He}$, as a function of the planet's heavy-element interior composition. Each point within the diagram corresponds to a specific combination of $\mathrm{Fe}, \mathrm{Mg}_{0.9} \mathrm{Fe}_{0.1} \mathrm{SiO}_{3}$, and $\mathrm{H}_{2} \mathrm{O}$ (by mass) in the heavy element interior of GJ 3470 b. For instructions on how to read ternary diagrams, see, e.g., Valencia et al. (2007) and Zeng \& Seager (2008). Each contour is labeled with $M_{\mathrm{XY}} / M_{p}$ for our fiducial model parameters (median $M_{p}$, median $R_{p}, A=0.3$, and $\left.L_{p} / M p=10^{-10} \mathrm{~W} \mathrm{~kg}^{-1}\right)$. Uncertainties in the planet mass, radius, and energy budget can affect $M_{\mathrm{XY}} / M_{p}$ by $\pm 0.03-0.05$.

GJ $1214 \mathrm{~b}$ are a remarkable sample of volatile-rich planets orbiting bright nearby stars, pushing the field of comparative exoplanetology further toward low-mass planets.

We thank Zach Berta and Elisabeth Newton for helpful discussions regarding near-IR M-dwarf spectral characterization. We are grateful to Rob Simcoe, Paul Schechter, Elisabeth Adams, and David Ciardi for their help in obtaining the ground-based observations presented in this paper. We thank the anonymous referee for a report that improved the paper. We thank the Spitzer Science Center staff, and especially Nancy Silbermann, for the efficient scheduling of our observations. We also wish to thank the staff of the Magellan Telescopes and Las Campanas Observatory for their assistance in obtaining the FIRE observations. This work is based on observations made with the Spitzer Space Telescope, which is operated by the Jet Propulsion Laboratory, California Institute of Technology, under a contract with NASA. Support for this work was provided by NASA through an award issued by JPL/Caltech. The WIYN Observatory is a joint facility of the University of Wisconsin-Madison, Indiana University, Yale University, and the National Optical Astronomy Observatory. Support for LAR was provided by NASA through Hubble Fellowship grant HF-51313.01-A awarded by the Space Telescope Science Institute, which is operated by the Association of Universities for Research in Astronomy, Inc., for NASA, under contract NAS 5-26555. This work was supported by the European Research Council/European Community under the FP7 through Starting Grant agreement number 239953 and by Fundação para a Ciência e a Tecnologia (FCT) in the form of grants PTDC/CTE-AST/098528/2008 and PTDC/CTE-AST/ $120251 / 2010$. The financial support from the "Programme National de Planétologie" (PNP) of CNRS/INSU, France, is grate- fully acknowledged. G.T. acknowledges partial support for this work through NSF grant AST-1007992. V.N. also acknowledges the support from the FCT in the form of the fellowship SFRH/BD/60688/2009. M.G. is Research Associate at the Belgian Fonds National de la Recherche Scientifique (FNRS).

Facilities: Spitzer, Magellan:Baade, WIYN

\section{REFERENCES}

Batalha, N. M., Rowe, J. F., Bryson, S. T., et al. 2013, ApJS, 204, 24 Bean, J. L., Kempton, E. M.-R., \& Homeier, D. 2010, Natur, 468, 669 Bonfils, X., Delfosse, X., Udry, S., et al. 2013, A\&A, 549, A109 Bonfils, X., Gillon, M., Udry, S., et al. 2012, A\&A, 546, A27 Boyajian, T. S., von Braun, K., van Belle, G., et al. 2012, ApJ, 757, 112 Butler, R. P., Vogt, S. S., Marcy, G. W., et al. 2004, ApJ, 617, 580 Carpenter, J. M. 2001, AJ, 121, 2851

Casagrande, L., Flynn, C., \& Bessell, M. 2008, MNRAS, 389, 585 Charbonneau, D., Berta, Z. K., Irwin, J., et al. 2009, Natur, 462, 891 Claret, A., \& Bloemen, S. 2011, A\&A, 529, 75

Cochran, W. D., Fabrycky, D. C., Torres, G., et al. 2011, ApJS, 197, 7 Delfosse, X., Forveille, T., Ségransan, D., et al. 2000, A\&A, 364, 217 Demory, B.-O., Gillon, M., Bonfils, X., et al. 2012a, Spitzer Proposal, 80261

Demory, B.-O., Gillon, M., Seager, S., et al. 2012b, ApJL, 751, L28

Dotter, A., Chaboyer, B., Jevremović, D., et al. 2008, ApJS, 178, 89 Eastman, J., Siverd, R., \& Gaudi, B. S. 2010, PASP, 122, 935

Evans, D. W., Irwin, M. J., \& Helmer, L. 2002, A\&A, 395, 347

Fazio, G. G., Hora, J. L., Allen, L. E., et al. 2004, ApJS, 154, 10

Figueira, P., Pont, F., Mordasini, C., et al. 2009, A\&A, 493, 671

Gelman, A., Carlin, J. B., Stern, H. S., \& Rubin, D. B. 2003, Bayesian Data Analysis (Chapman \& Hall/CRC Texts in Statistical Science, 2nd ed.; London: Chapman and Hall)

Gelman, A., \& Rubin, D. B. 1992, StaSc, 7, 457

Gillon, M., Lanotte, A. A., Barman, T., et al. 2010, A\&A, 511, 3

Gillon, M., Pont, F., Demory, B.-O., et al. 2007, A\&A, 472, L13

Hartman, J. D., Bakos, G. Á., Kipping, D. M., et al. 2011, ApJ, 728, 138

Holman, M. J., Winn, J. N., Latham, D. W., et al. 2006, ApJ, 652, 1715

Horch, E. P., Howell, S. B., Everett, M. E., \& Ciardi, D. R. 2012, AJ, 144, 165

Horch, E. P., Veillette, D. R., Baena Gallé, R., et al. 2009, AJ, 137, 5057 
Howard, A. W., Marcy, G. W., Bryson, S. T., et al. 2012, ApJS, 201, 15 Howard, A. W., Marcy, G. W., Johnson, J. A., et al. 2010, Sci, 330, 653 Ingalls, J. G., Krick, J. E., Carey, S. J., et al. 2012, Proc. SPIE, 8442, 1

Kennedy, G. M., Kenyon, S. J., \& Bromley, B. C. 2007, Ap\&SS, 311, 9

Kervella, P., Thévenin, F., Di Folco, E., \& Ségransan, D. 2004, A\&A, 426, 297

Knutson, H. A., Lewis, N., Fortney, J. J., et al. 2012, ApJ, 754, 22

Lissauer, J. J. 2007, ApJL, 660, L149

Lissauer, J. J., Fabrycky, D. C., Ford, E. B., et al. 2011, Natur, 470, 53

Mann, A. W., Brewer, J. M., Gaidos, E., Lepine, S., \& Hilton, E. J. 2013, AJ, 145,52

Neves, V., Bonfils, X., Santos, N. C., et al. 2012, A\&A, 538, A25

Neves, V., Bonfils, X., Santos, N. C., et al. 2013, A\&A, 551, A36

Nutzman, P., \& Charbonneau, D. 2008, PASP, 120, 317

Ogihara, M., \& Ida, S. 2008, in IAU Symp. 249, Exoplanets: Detection, Formation and Dynamics, ed. Y.-S. Sun, S. Ferraz-Mello, \& J.-L. Zhou (Cambridge: Cambridge Univ. Press), 305

Pineda, J. S., Bottom, M., \& Johnson, J. A. 2013, arXiv:1302.6231

Rogers, L. A., Bodenheimer, P., Lissauer, J. J., \& Seager, S. 2011, ApJ, 738, 59
Rogers, L. A., \& Seager, S. 2010a, ApJ, 712, 974

Rogers, L. A., \& Seager, S. 2010b, ApJ, 716, 1208

Rojas-Ayala, B., Covey, K. R., Muirhead, P. S., \& Lloyd, J. P. 2012, ApJ, 748, 93

Seager, S., Kuchner, M., Hier-Majumder, C. A., \& Militzer, B. 2007, ApJ, 669, 1279

Seager, S., \& Mallén-Ornelas, G. 2003, ApJ, 585, 1038

Simcoe, R. A., Burgasser, A. J., Bernstein, R. A., et al. 2008, Proc. SPIE, 7014 $70140 \mathrm{U}$

Stevenson, K. B., Harrington, J., Nymeyer, S., et al. 2010, Natur, 464, 116

Terrien, R. C., Mahadevan, S., Bender, C. F., et al. 2012, ApJL, 747, L38

Torres, G. 2013, AN, 334, 4

Torres, G., Winn, J. N., \& Holman, M. J. 2008, ApJ, 677, 1324

Vacca, W. D., Cushing, M. C., \& Rayner, J. T. 2003, PASP, 115, 389

Valencia, D., Sasselov, D. D., \& O'Connell, R. J. 2007, ApJ, 665, 1413

Weis, E. W. 1986, AJ, 91, 626

Wittenmyer, R. A., Tinney, C. G., Butler, R. P., et al. 2011, ApJ, 738, 81

Zacharias, N., Finch, C. T., Girard, T. M., et al. 2013, AJ, 145, 44

Zeng, L., \& Seager, S. 2008, PASP, 120, 983 